\title{
A Photochemical Crosslinking Technology for Tissue Engineering - Enhancement of the Physico-Chemical Properties of Collagen-based Scaffolds
}

\author{
B.P. Chan, Ph.D. \\ bpchan@hkucc.hku.hk \\ Research Assistant Professor, Medical Engineering Program, The University of Hong Kong, \\ Pokfulam Road, Hong Kong Special Administrative Region, China. \\ Tel.: (852) 2857 8553, Fax: (852) 28585415
}

\begin{abstract}
Collagen gel is a natural biomaterial commonly used in tissue engineering because of its close resemblance to nature, negligible immunogenecity and excellent biocompatibility. However, unprocessed collagen gel is mechanically weak, highly water binding and vulnerable to chemical and enzymatic attacks that limits its use in tissue engineering in particular tissues for weightbearing purposes.
\end{abstract}

The current project aimed to strengthen and stabilize collagen scaffolds using a photochemical crosslinking technique. Photochemical crosslinking is rapid, efficient, non-thermal and does not involve toxic chemicals, comparing with other crosslinking methods such as glutaraldehyde and gamma irradiation.

Collagen scaffolds were fabricated using rat-tail tendon collagen. An argon laser was used to process the collagen gel after equilibrating with a photosensitizing reagent. Scanning electronic microscope was used to characterize the surface and cross-sectional morphology of the membranes. Physico-chemical properties of the collagen scaffolds such as water-binding capacity, mechanical properties and thermostability were studied.

Photochemical crosslinking significantly reduced the water-binding capacity, a parameter inversely proportional to the extent of crosslinking, of collagen scaffolds. Photochemical crosslinking also significantly increased the ultimate stress and tangent modulus at $90 \%$ of the rupture strain of the collagen scaffolds. Differential scanning calorimetry analysis showed a significantly higher shrinkage temperature and absence of the denaturation peak during the thermoscan comparing with the controls. This means greater thermostability in the photochemically crosslinked collagen scaffolds.

This study demonstrates that the photochemical crosslinking technology is able to enhance the physicochemical properties of collagen scaffolds by strengthening, stabilizing and controlling the swelling ratio of the collagen scaffolds so as to enable their use for tissue engineering.

Key words:

Photochemical crosslinking, collagen, physicochemical properties 


\section{Introduction}

Collagen is the most abundant and ubiquitous protein in human body ( $\mathrm{Li} \mathrm{2000)}$ ) and is a natural biomaterial commonly used in tissue engineering because of its close resemblance to the nature and negligible immunogenecity (Yannas 1996). However, the use of collagen as scaffolds in engineered tissues in particular those for weight bearing, is limited because untreated collagen is weak, with low processability, easy to collapse owing to its vulnerability towards chemical and enzymatic attacks (Yannas 1996).

Crosslinking methods, either chemical or physical (Khor 1997), have been used to improve the properties of collagen but are of significant limitations. Glutaraldehyde is the most efficient chemical crosslinking reagent for proteins (Charulatha \& Rajaram 2002). However, use of glutaraldehyde induces cytotoxicity and calcification in host tissue (Khor 1997, Simmons \& Kearney 1993, Lee et al. 2001). This seriously compromises the biocompatibility of the scaffolds. Physical methods such as dehydrothermal treatment (DHT), UV-and $\gamma$-irradiation have been used. However, these methods are time-consuming and induce collagen denaturation that compromises the scaffold stability (Pieper et al. 1999, Weadock et al. 1995, Billiar et al. 2001). As a result, alternative methods improving the physicochemical properties of collagen gel without compromising their biocompatibility and stability are needed.

Photochemical reactions of soluble proteins such as fibrinogen (Webster et al 1989) and lens protein crystallins (Balasubramanian D et al. 1990) in the presence of light and photosensitizing reagents have been demonstrated in last decade. It has been suggested that some amino acid groups such as tryptophan, tyrosine, histidine, cysteine and methionine are vulnerable to photochemical crosslinking (Balasubramanian et al. 1990, Hott \& Borkman 1992) although it has also been proposed that the crosslinking in proteins is non-specific and the actual crosslinking sites could not be accurately located (Hott \& Borkman 1992). However, to our knowledge, there is no published report using photochemical crosslinking for processing proteinous biomaterials such as collagen gel. In contrast to chemical and physical methods, the photochemical crosslinking method has been shown to form crosslinks rapidly, works under ambient temperature far below the denaturation temperatures for collagen, without usage of toxic chemicals and does not compromise cell viability (Chan et al. 2002, Chan et al. 2004).

The current project aims to investigate the potential of using photochemical crosslinking to modify the physicochemical properties of collagen gel in order to enable its use in tissue engineering.

\section{Methodology}

\section{$\underline{\text { Reconstitution of collagen }}$}

Rattail collagen type I solution (Becton, Dickinson, MA) at a concentration of $4 \mathrm{mg} / \mathrm{ml}$ was used. Collagen solution was laid down onto 4-well culture plates (Nunclon, Denmark) with $1.5 \mathrm{ml}$ per well and exposed to an ammonia chamber for 30 minutes. The reconstituted gel was $1.5 \mathrm{~cm}$ in diameter.

\section{Photochemical crosslinking of reconstituted collagen gel}

The reconstituted collagen gel was equilibrated with $10 \mathrm{ml}$ of photosensitizing reagent, rose Bengal (Sigma, St. Louis) at a concentration of $0.01 \%(\mathrm{w} / \mathrm{v})$ in distilled water for 2 hours with constant 
agitation in darkness. An argon laser (Ultima 2000, Coherent Medical Group, Santa Clara) with a spot size of $1.5 \mathrm{~cm}$ in diameter was delivered in pulses at a power of $0.2 \mathrm{~W}$ with duration of $1 \mathrm{~s}$. A total of 125 pulses equivalent to $25 \mathrm{~J}$ was used for photochemical crosslinking. Untreated collagen gel was used as the control. Dye control and laser control groups were collagen gels treated with the photosensitizing reagent alone and laser irradiation at the highest dosage alone, respectively. Glutaraldehyde was used as positive control. All collagen gels were air-dried completely.

\section{$\underline{\text { Scanning Electron Microscope (SEM) analysis }}$}

Dry collagen membranes were mounted on metal stabs using carbon adhesive tapes and were critical point-dried. The membranes were sputtered with gold and analyzed using a SEM (Cambridge SEM 440) for characterization of the surface and cross-sectional morphology.

\section{Measurement of the swelling ratio}

Collagen membranes were weighed to obtain the dry weight $\left(\mathrm{W}_{\mathrm{d}}\right)$. Membranes with 2-3mg of dry weight were thoroughly rinsed with distilled water and rehydrated for measurement of the swelling ratio. The wet weight of the rehydrated collagen membranes $\left(W_{w}\right)$ after blotting the surface water was measured at 96 hours after rehydration. The swelling ratio of the collagen membranes was calculated as follows:

$$
\text { Swelling ratio }=\left(\mathrm{W}_{\mathrm{w}}-\mathrm{W}_{\mathrm{d}}\right) /\left(\mathrm{W}_{\mathrm{d}}\right)
$$

Four collagen membranes were used in each treatment group.

\section{Measurement of the mechanical properties}

Collagen membranes were rehydrated in phosphate buffered saline (PBS) at $\mathrm{pH} 7.4$ for more than 24 hours and were carved into a dumpbell shape with a width of $3 \mathrm{~mm}$ and a gauge length of $6 \mathrm{~mm}$ using a custom-made punch. The dimensions of the collagen membranes were measured using a Quick Vision QVPro202 system (Mitutoyo). A tensile testing machine (LR50K, Lloyd Instruments, UK) connected to a load cell of $10 \mathrm{~N}$ with $0.5 \%$ accuracy was used. A uniaxial force at a constant strain rate of $5 \mathrm{~mm} / \mathrm{min}$ was applied to the collagen membrane and the force and displacement were recorded. A CCD camera was used for strain measurement at a rate of 25 frames per second. Stress-strain curves were obtained and the mechanical properties of the collagen membranes, including the ultimate stress and tangent modulus at $90 \%$ of the rupture strain were calculated. Two to three collagen membranes were used in each of the treatment groups.

\section{Measurement of the thermo-stability}

Collagen membranes with a dry weight of 1-3 mg were rehydrated in PBS ( $\mathrm{pH} 7.4$ ) at room temperature for more than 24 hours. Surface water of the collagen membranes was removed before the thermal analysis using a differential scanning calorimeter (DSC7.0, Perkin Elmer, Boston). The samples were hermetically sealed in aluminium pans and placed in the sample holding stage while a reference pan without sample was used as the control. Thermoscans from $25^{\circ} \mathrm{C}$ to $140^{\circ} \mathrm{C}$ at a rate of $5^{\circ} \mathrm{C} / \mathrm{min}$ were performed. Pyris Manager software (Perkin Elmer) was used to measure the shrinkage temperature $\left(T_{s}\right)$. Remnants of the samples after the thermoscan were rehydrated in the same buffer and fixed in $0.02 \%$ glutaraldehyde for 4 hours at room temperature. The thermally treated remnants were processed for SEM analysis. 
$\underline{\text { Statistics and data analysis }}$

Data obtained were all continuous measurements and were verified with the assumption for normal distribution. Natural log transformation was performed if necessary. One-way ANOVA with Bonferroni's post-hoc tests were used to compare the difference among different treatment groups. Kruskal Wallis test was used if the sample was distribution-free. SPSS 12.0 was used to execute all data processing and analysis and the significance level was set at 0.05 .

\section{Results}

\section{$\underline{\text { Surface and cross-sectional morphology }}$}

Collagen membranes in different treatment groups did not differ significantly from one another on the surface and cross-sectional morphology. They all showed relatively smooth surfaces and form well-organized lamellae-like structures at the cross-sections (Fig. 1A). Well-organized structures with orientations in the collagen membranes were also observed (Fig. 1B).

\section{$\underline{\text { Swelling ratios }}$}

Fig. 2 showed the box plot of the swelling ratio of collagen membranes at equilibrium in different treatment groups. The positive control group glutaraldehyde showed a very low swelling ratio at around 0.6 with a water content of $\sim 38 \%$. The untreated control group showed a swelling ratio of 6.3 with water content around $86 \%(\mathrm{w} / \mathrm{w})$. Comparing with the untreated control, the laser control group showed a higher swelling ratio at 7.2 with $\sim 88 \%$ water content $(\mathrm{w} / \mathrm{w})$ while the dye control group showed a lower swelling ratio at 4.8 that the water content was around $83 \%$. Photochemically crosslinked groups at different dosages showed an intermediate swelling ratio ranging from 2.7 to 4.2 giving a range of water content from $\sim 70$ to $80 \%(\mathrm{w} / \mathrm{w})$. One-way ANOVA showed that there was statistically significant difference among different treatment groups $(p<0.001)$. Bonferroni's post-hoc tests showed that all photochemically crosslinked groups were significantly different in swelling ratio than the untreated, the laser and the positive controls $(\mathrm{p}<0.001)$ while all but the lowest laser dosage group $(12.5 \mathrm{~J})$ showed significant difference with the dye control $(\mathrm{p}<=0.003)$. As for different laser dosage groups, a significant negative linear trend was detected $(\mathrm{p}<0.001)$.

Mechanical properties

Fig. 3 showed the representative stress-strain curves of collagen membranes in different treatment groups. Untreated control and laser control groups showed the most shallow stress-strain relationship that very low stress was found with large increase in strain and they failed at very high strain. The dye control group showed a much greater rate of increase in stress as the samples were strained and failed at less than $20 \%$ strain. Photochemically crosslinked collagen membranes in different dosage groups showed even steeper increases in stress as the strain increased. The positive control showed the greatest rate of increase in stress as it was strained. Fig. 4 showed the box plot of the ultimate stress of collagen membranes in different groups. The control groups showed median ultimate stress well below $1 \mathrm{MPa}$ while the photochemically crosslinked ones showed at least two fold changes comparing with the controls and the positive control glutaraldehyde showed median value close to $4 \mathrm{MPa}$. Kruskal Wallis test showed that there was significant difference among the groups $(\mathrm{p}=0.033)$. Fig. 5 showed the tangent modulus of collagen membranes at $90 \%$ rupture strain. The untreated and the laser controls showed very low tangent 
modulus at $90 \%$ rupture strain that the median values were around $1 \mathrm{MPa}$ while the photochemically crosslinked ones showed 10-20 fold changes that the median values ranged from around 10 to $20 \mathrm{MPa}$. The dye control showed a small increase in tangent modulus comparing with the other controls that the median value was close to $8 \mathrm{MPa}$. The positive control showed median tangent modulus above $40 \mathrm{MPa}$. Kruskal Wallis test showed significance difference among the groups $(\mathrm{p}=0.026)$.

\section{Thermo-stability}

Fig. 6 showed the representative thermograms of collagen membranes in various control groups (A) and crosslinked groups (B). In all control groups, the shrinkage temperature peaks were found between 70 and $90^{\circ} \mathrm{C}$ and characterized by a significant shrinkage in volume of the fully swollen collagen membranes. All control groups were then undergoing an endothermic event of denaturation or "solubilization" process at temperatures around $100-120^{\circ} \mathrm{C}$ where multiple numbers of endothermic peaks were found. As for all crosslinked groups, single endothermic peak representing the shrinkage temperature peak was found well above $90^{\circ} \mathrm{C}$ to around $110^{\circ} \mathrm{C}$ and the thermoscans of the crosslinked groups were characterized by the absence of the denaturation or "solubilization" peaks throughout the thermoscan up to $140^{\circ} \mathrm{C}$. This was demonstrated by the fact that solid collagen membrane, although shrank in volume, were found in the aluminium pans at the end of the thermoscan in all crosslinked groups. These post-thermoscan remnants of the photochemically crosslinked collagen membranes were able to regain their 3-D structure after rehydration as shown in the SEM pictures (Fig. 7B) while the control groups completely lost the 3D structure (Fig. 7A). Fig. 8 summarized the shrinkage temperatures of collagen membranes in different treatment groups. Eight samples were used in each treatment group. One-way ANVOA showed significant differences among the treatment groups $(\mathrm{p}<0.001)$. Post-hoc Bonferroni's tests showed that all photochemically crosslinked collagen membranes had significantly higher shrinkage temperatures than the controls $(p<=0.004)$ and laser dosage groups from $6.25 \mathrm{~J}$ to $50 \mathrm{~J}$ were significantly greater than the dye control groups $(\mathrm{p}<=0.012)$ while the chemically crosslinked collagen membranes (glutaradehyde) did not show significant difference from the control groups.

\section{Discussion}

This study demonstrated that the photochemical crosslinking technology, with numerous advantages over the existing chemical or physical crosslinking methods, is able to improve the physicochemical properties of collagen-based scaffolds for tissue engineering purposes.

Mechanical properties are crucial parameters in designing collagen-based scaffolds for tissue engineering in particular tissues for weight-bearing purpose. The photochemical crosslinking technology has significantly strengthen and stiffen the collagen scaffolds through modifications of their stress-strain relationship that the photochemically crosslinked collagen scaffolds tend to have a more rapid increase in stress as it strains, a greater tangent modulus at the same percentage of rupture strain comparing with the uncrosslinked controls. Although glutaraldehyde showed higher ultimate stress and tangent modulus than the photochemically crosslinked ones, its use is limited by its toxic residues and metabolites which induce cytotoxicity and calcification upon implantation (Simmons \& Kearney 1993, Lee et al. 2001). Other crosslinking agents under investigations include chemical agent carbodiimide derivatives and natural crosslinking reagent genipin. However, both of them only reported minor improvement on the mechanical properties (Angele et al. 2004, Bigi et al. 2002). 
Photochemical crosslinking has been shown to chemically and thermally stabilize the collagenbased scaffolds tremendously. This indicates that the nature of photochemical crosslinking is covalent and the intermolecular linkages formed are thermostable. On the other hand, the uncrosslinked collagen membranes denatured and lost their tertiary and secondary structures to give amorphous structure of gelatin as temperature increased. Moreover, a higher shrinkage temperature in photochemical crosslinked scaffolds also indicate that photochemical crosslinking stabilizes the intramolecular hydrogen linkages so that binding water to these sites become more thermostable. These improved features may assure stable storage of collagen-based scaffolds even at a swollen state and at temperatures well above the normal storage conditions and may also enable further treatments or processes of the collagen-based scaffolds with harsh conditions in temperature and pressure while this is impossible in uncrosslinked controls.

Collagen gel is highly water swollen that unprocessed control collagen gel has been found with more than $86 \%$ water in fully swollen status. The swelling ratio measuring the water binding capacity of a hydrogel usually has an inverse relationship with the extent of crosslinking. Swelling is a favorable event in tissue engineering where new tissue growth should be facilitated. Swelling is also one of the mechanisms for biomolecules such as growth factors immobilized within the scaffolds to be released. Thus, swollen matrices may also provide means for drug delivery at the local site. In the current study, photochemically crosslinked collagen scaffolds have been found with intermediate level of swelling ratios comparing with the glutaradehyde and the control groups and are dependent on the amount of radiation energy. As a result, it is possible to control the swelling properties and hence the controlled release rate of drugs immobilized within the collagen matrix by controlling the extent of photochemical crosslinking.

The current study also showed that both the laser and the dye control have significant effects on the mechanical properties and the swelling properties of the scaffolds. Laser control seems to disrupt the intermolecular and intramolecular bonding in collagen scaffolds permanently. This may be due to the fact that biomolecules such as amino acids are able to absorb visible lights that these groups may be chemically altered and the native bonding in collagen scaffolds may be affected. On the other hand, these vulnerable amino acids or groups may be "protected" against laser damage when the photosensitizing reagent are bound to these exposed groups in collagen scaffolds as in the dye control group of the current study. The photosensitizing reagent may bind to these vulnerable groups and interacting with the intra-molecular hydrogen linkages that stabilizes the scaffold. Nevertheless, the stabilizing effects of the dye control group are due to non-covalent interactions and therefore disappear upon thermal challenge. Further, the weak stabilizing effect of the photosensitizing reagent may also be due to the inevitable exposure of the dye control scaffolds to diffuse light during experimental procedures such as measuring the dimension of the scaffolds using optical methods.

\section{Acknowledgement}

This work was partially supported by University Research Committee, the University of Hong Kong (No. 10205248). The authors thank Ms. Pandora Yip and Ms. Carrie Wong for assistance with the data analyses, and Ms. Joean Kwok for assistance with the DSC analysis. 


\section{References}

1. Angele P, Abke J, Kujat R, Faltermeier H, Schumann D, Nerlich M, Kinner B, Englert C, Ruszczak Z, Mehrl R, Mueller R. (2004) Influence of different collagen species on physicochemical properties of crosslinked collagen matrices. Biomaterials. 25(14):2831-41.

2. Balasubramanian D, Du X, Zigler JS Jr. (1990) The reaction of singlet oxygen with proteins, with special reference to crystallins. Photochem Photobiol. 52(4):761-8.

3. Bigi A, Cojazzi G, Panzavolta S, Roveri N, Rubini K. (2002) Stabilization of gelatin films by crosslinking with genipin. Biomaterials. 23(24):4827-32.

4. Billiar K, Murray J, Laude D, Abraham G, Bachrach N. (2001) Effects of carbodiimide crosslinking conditions on the physical properties of laminated intestinal submucosa. $\mathrm{J}$ Biomed Mater Res. 56(1):101-8.

5. Chan BP et al. (2004) A light-activated tissue bonding in tendon repair - ex vivo and in vivo study. (J Surg Res, in print)

6. Chan BP, Kochevar IE, Redmond RW. (2002) Enhancement of porcine skin graft adherence using a light-activated process. J Surg Res.108(1):77-84.

7. Charulatha V, Rajaram A. (2003) Influence of different crosslinking treatments on the physical properties of collagen membranes. Biomaterials. 24(5):759-67.

8. Hott JL, Borkman RF. (1992) Analysis of photo-oxidized amino acids in tryptic peptides of calf lens gamma-II crystallin. Photochem Photobiol. 56(2):257-63.

9. Khor E. (1997) Methods for the treatment of collagenous tissues for bioprostheses. Biomaterials. 1997 Jan;18(2):95-105.

10. Lee JE et al. (2001) Characterization of UV-irradiated dense/porous collagen membranes: morphology, enzymatic degradation, and mechanical properties. Yonsei Med J. 2001 Apr;42(2):172-9.

11. Li ST. (2000) Biologic biomaterials: Tissue-derived biomaterials (collagen). In The Biomedical Engineering Handbook, $2^{\text {nd }}$ ed. Bronzino JD (ed.), Boca Raton: CRC Press LLC. Ch.4.

12. Pieper JS, Oosterhof A, Dijkstra PJ, Veerkamp JH, van Kuppevelt TH. (1999) Preparation and characterization of porous crosslinked collagenous matrices containing bioavailable chondroitin sulphate. Biomaterials. 1999 May;20(9):847-58.

13. Simmons DM, Kearney JN. (1993) Evaluation of collagen cross-linking techniques for the stabilization of tissue matrices. Biotechnol Appl Biochem. 17 (1):23-9.

14. Weadock KS et al. (1995) Crosslinking of collagen fibers: comparison of ultraviolet irradiation and dehydrothermal treatment. J Biomed Mater Res 29(11):1373-9.

15. Webster A, Britton D, Apap-Bologna A, Kemp G. (1989) A dye-photosensitized reaction that generates stable protein-protein crosslinks. Anal Biochem. 179(1):154-7.

16. Yannas IV Natural materials. In Ratner BD, Hoffman AS, Schoen FJ, Lemons JE (eds). Biomaterials Sciences - An introduction to materials in medicine. Academic Press, 1996, California, USA. Pp84-93. 


\section{Pictures}

Fig. 1 - SEM pictures showing the surface and cross-sectional morphology of collagen membranes
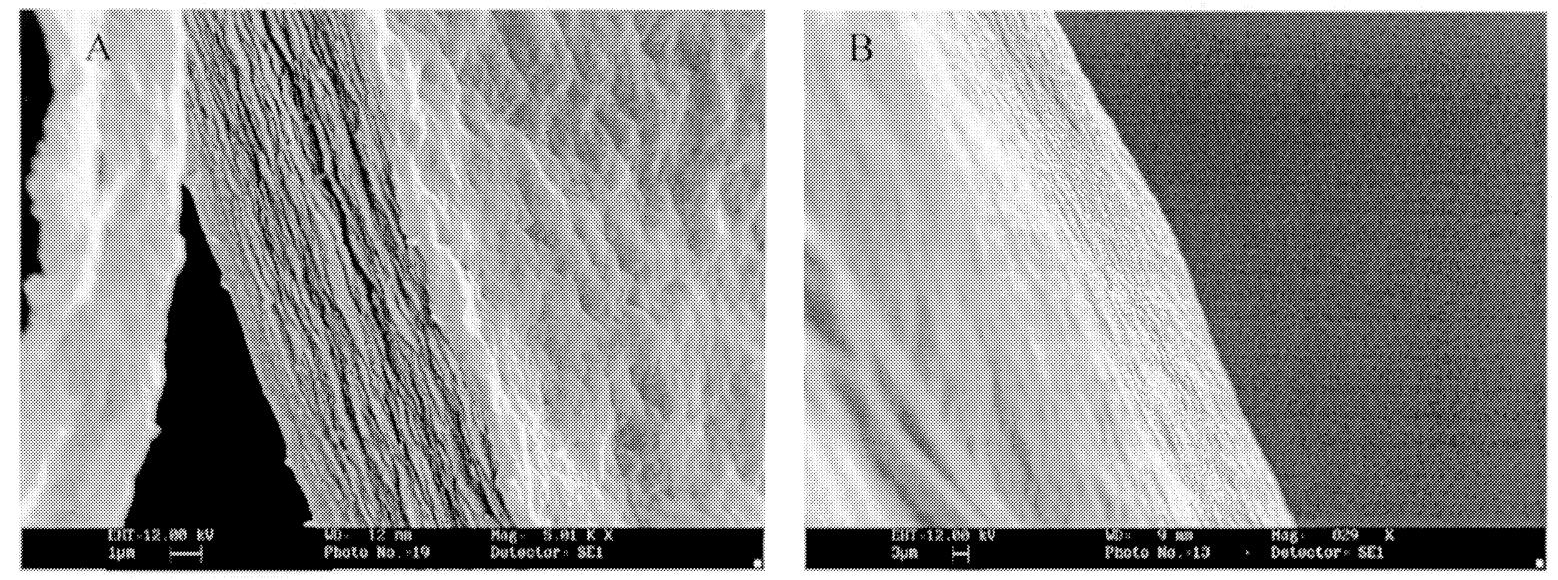

Fig. 2 - Box plot of the swelling ratio of collagen membranes in different treatment groups

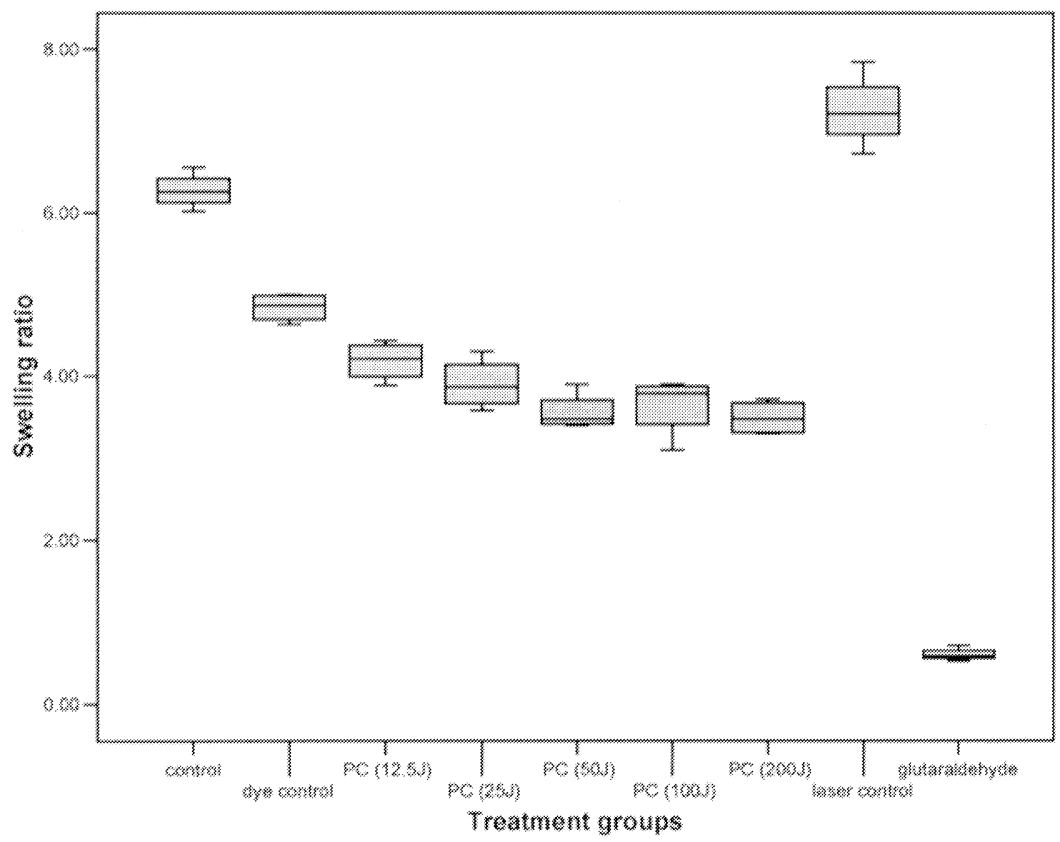


Fig. 3 - Representative stress-strain curves of collagen membranes in different treatment groups

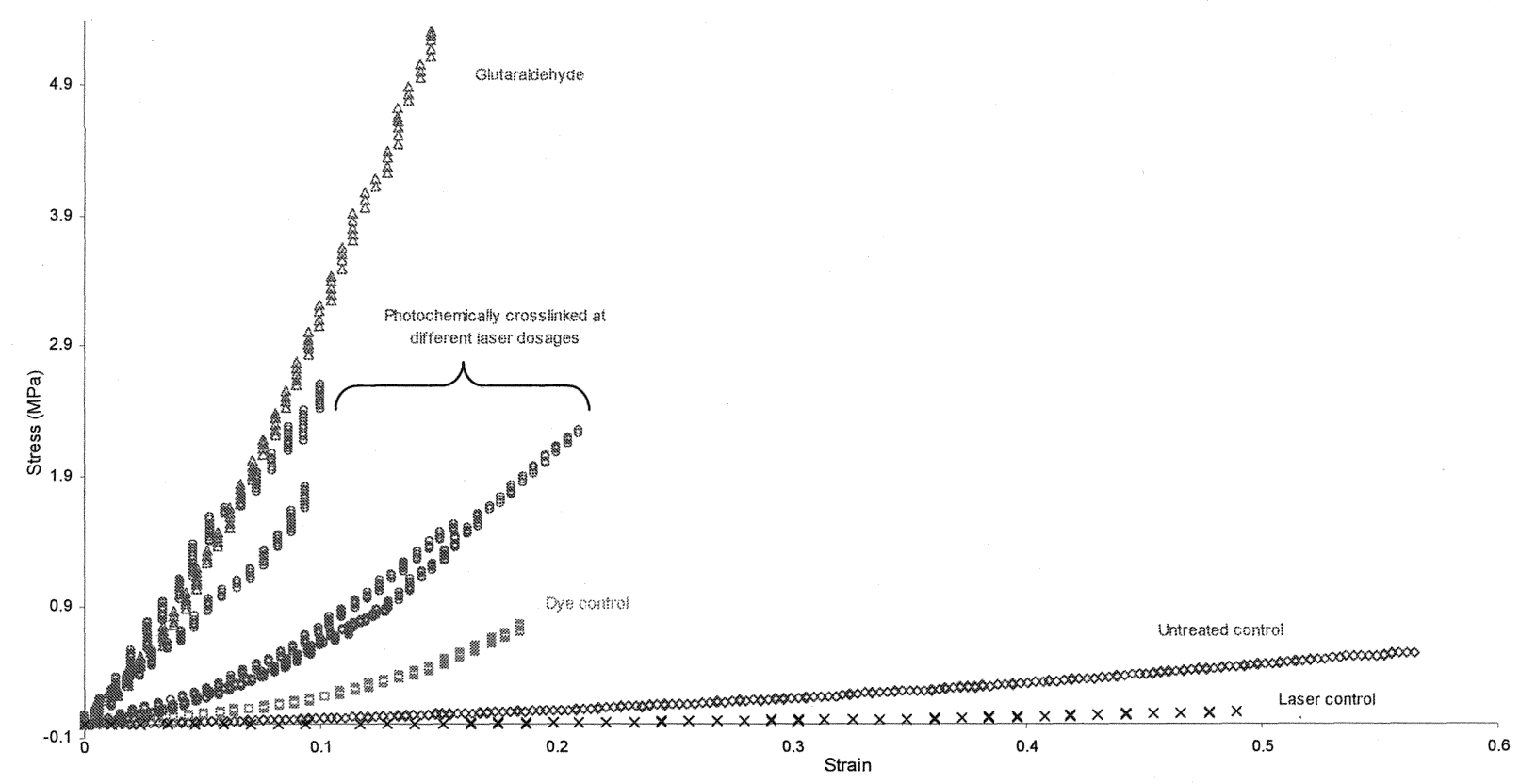

Fig. 4 - Box plot of the ultimate stress of the collagen membranes in different treatment groups

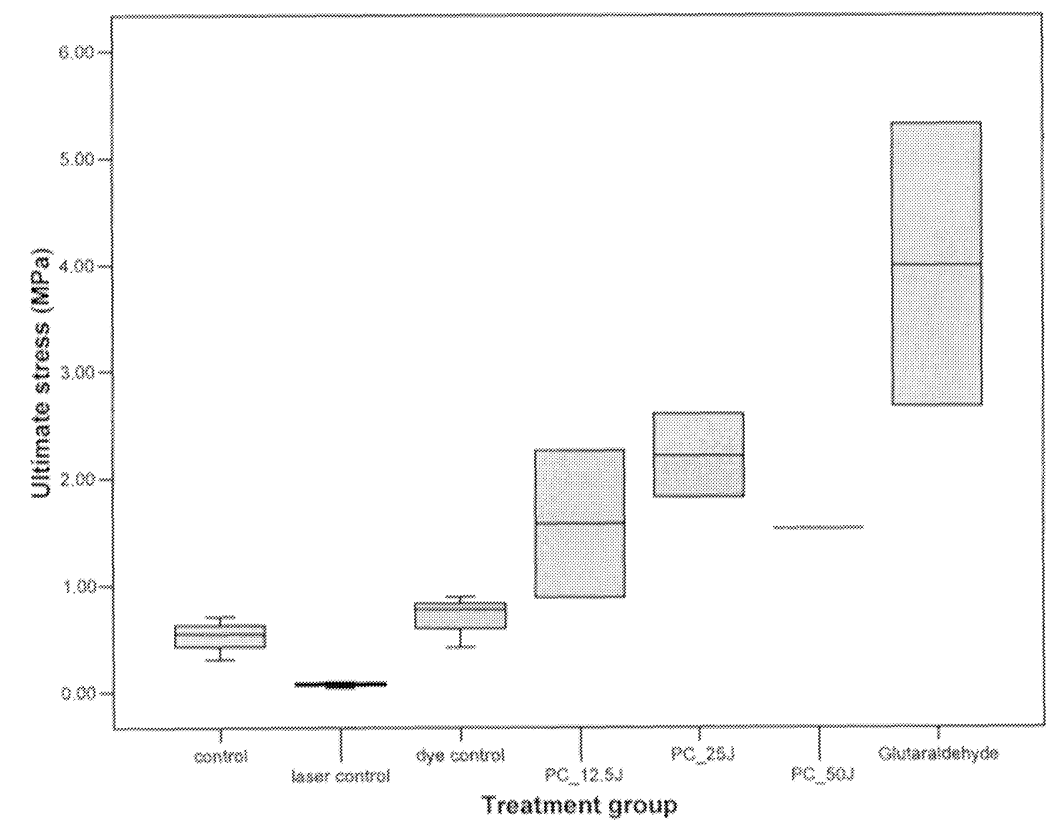


Fig. 5 - Box plot of the tangent modulus at $90 \%$ rupture strain of collagen membranes in different treatment groups

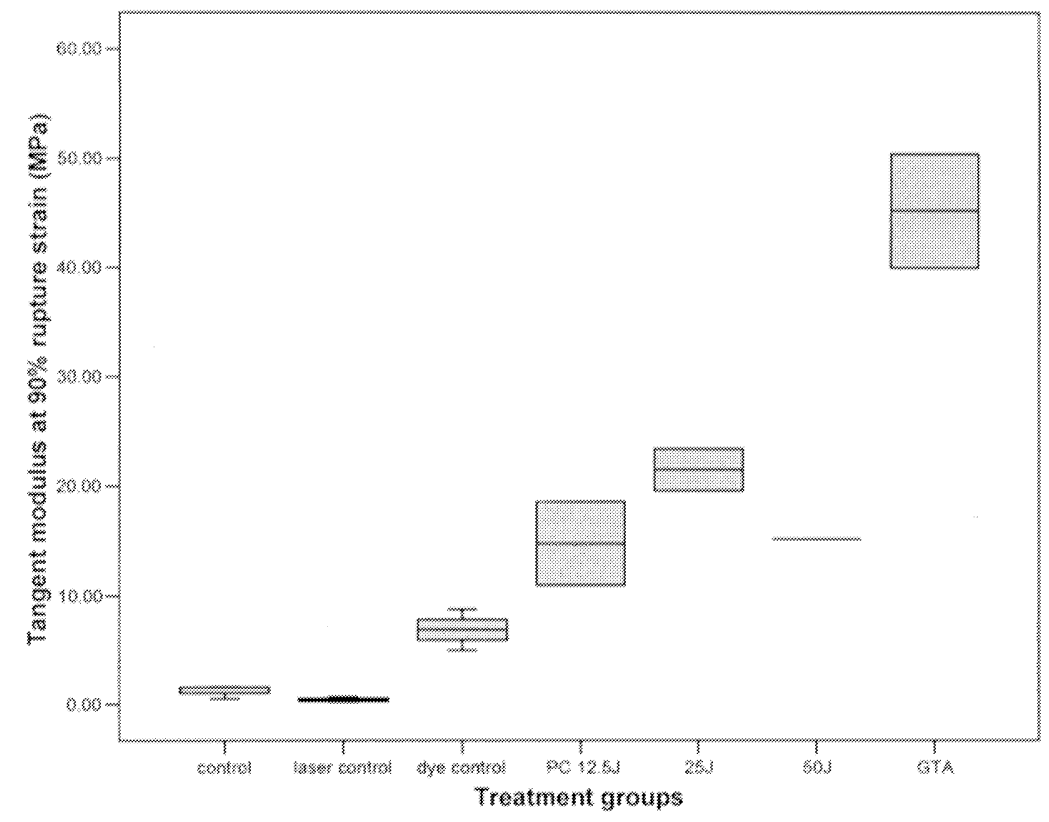

Fig. 6 - Representative thermograms (A-controls; B-crosslinked)
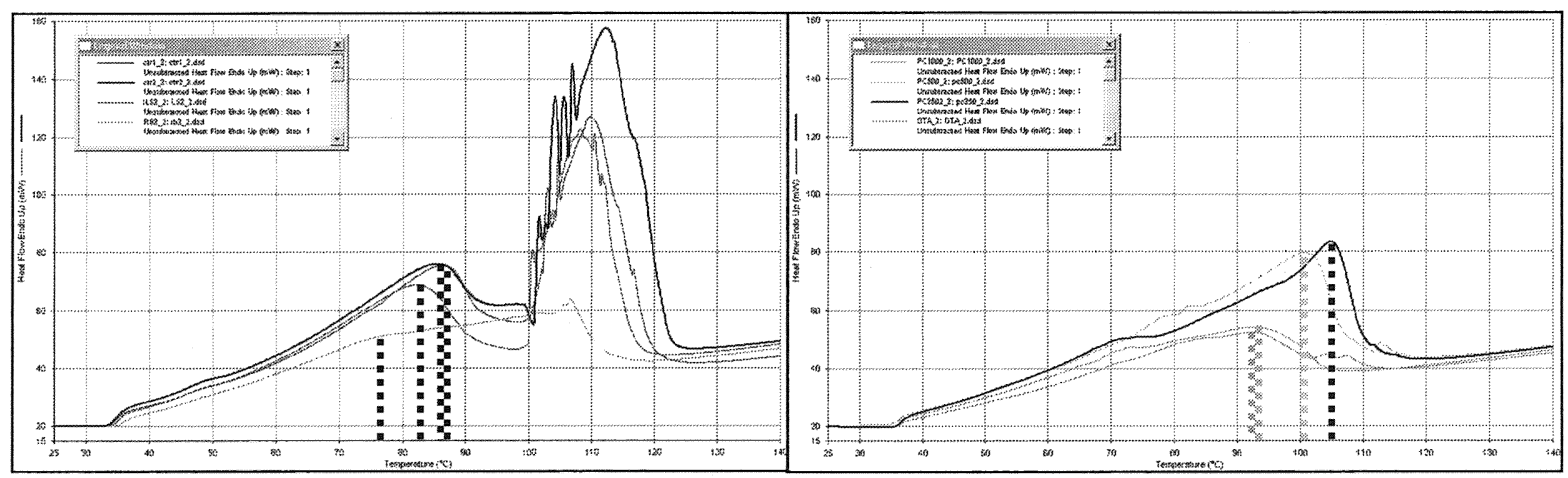
Fig. 7 - SEM pictures of the remnants of the collagen scaffolds after the thermoscan . (A) photochemically crosslinked scaffold; (B): Control scaffold. (Mag. 1KX)
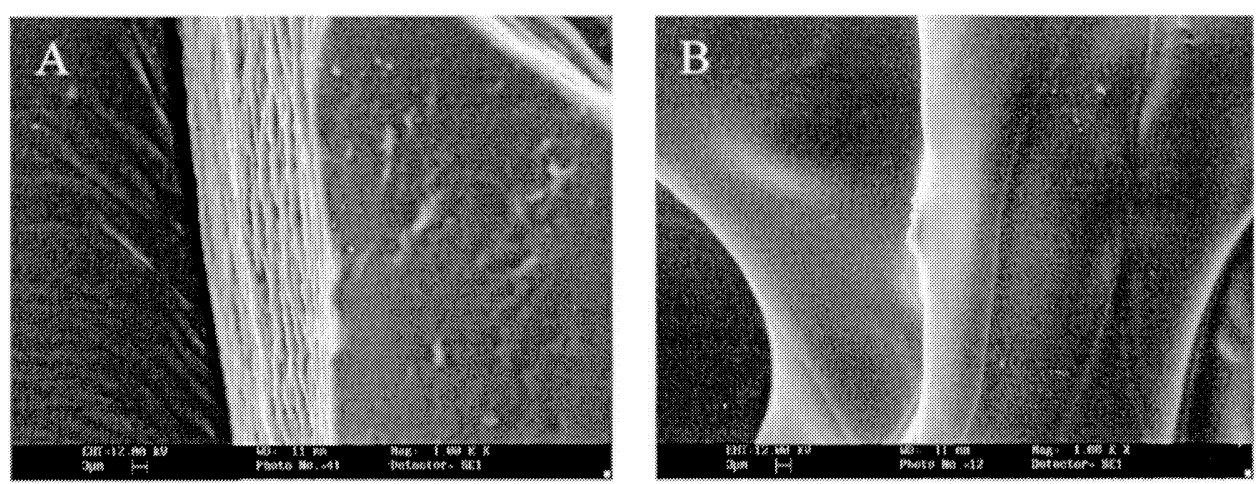

Fig. 8 - Box plot of shrinkage temperature of collagen membranes in different treatment groups

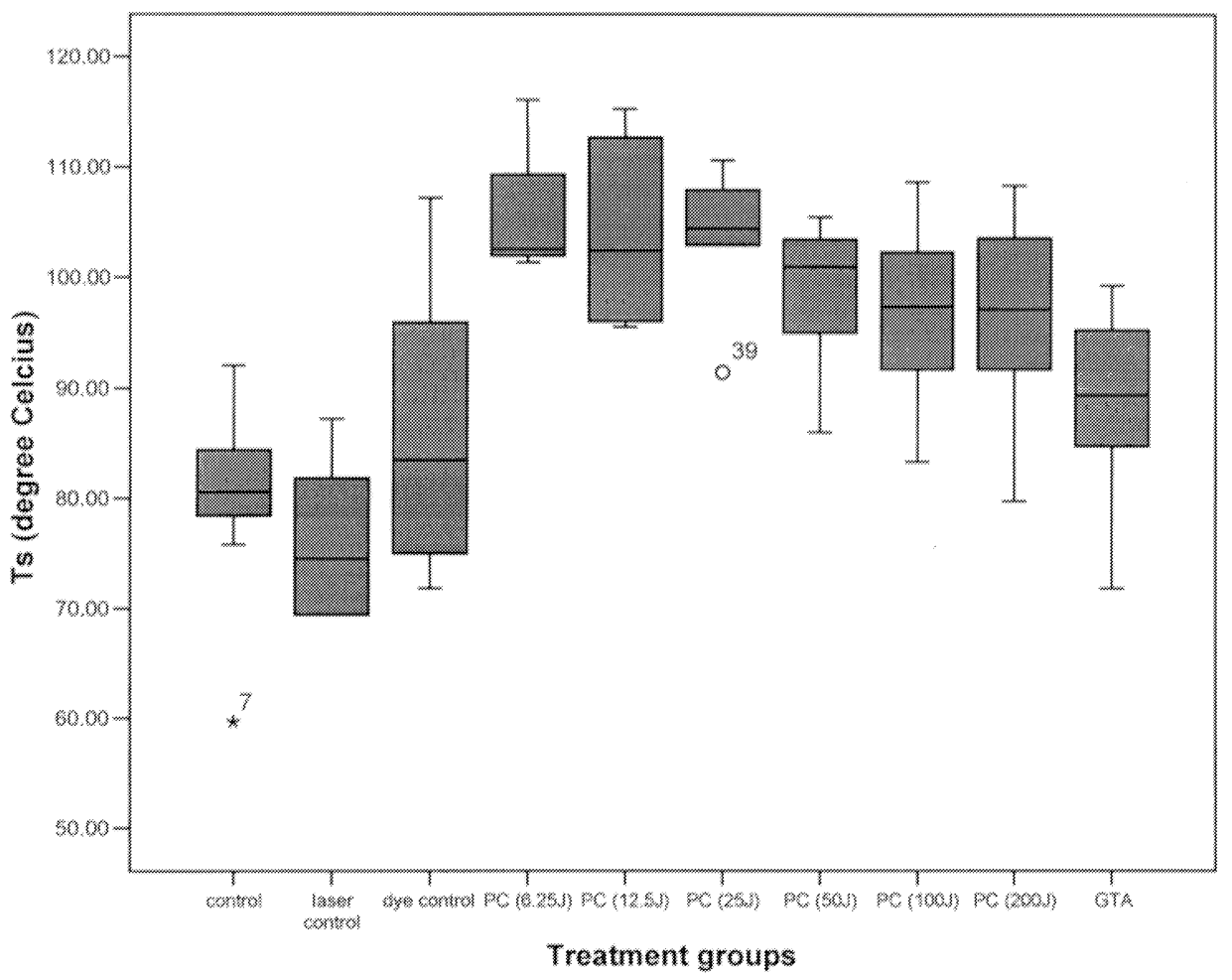

